\title{
miR-30a regulates the proliferation and invasion of breast cancer cells by targeting Snail
}

\author{
BAOQIANG XIAO $^{1^{*}}, \mathrm{XUEJING} \mathrm{SHI}^{2^{*}}$ and JIANPING BAI \\ ${ }^{1}$ Department of General Surgery, No. 254 Hospital of The People's Liberation Army; \\ ${ }^{2}$ Department of Breast Surgery, Tianjin Central Obstetrics and Gynecology Hospital, Tianjin 300142, P.R. China
}

Received December 12, 2016; Accepted February 7, 2018

DOI: $10.3892 / \mathrm{ol} .2018 .9552$

\begin{abstract}
The present study aims to investigate the effect of miR-30a on the proliferative and invasive abilities of breast cancer cells, and to observe the role of miR-30a in the pathogenesis of breast cancer. With the increase of pathological grade and malignant degree of breast cancer cells, the miR-30a expression level gradually decreased $(\mathrm{P}<0.01)$. Transfection with miR-30a mimic significantly inhibited the proliferative and invasive ability of SK-BR-3 cells $(\mathrm{P}<0.01)$, while transfection with anti-miR-30a significantly improved the proliferative and invasive ability of these cells $(\mathrm{P}<0.01)$. It was revealed using bioinformatic methods that Snail was the functional target gene of miR-30a, and this was verified by the results of a luciferase reporter gene assay. The results of analysis of Snail expression in breast cancer tissues and breast cancer cells revealed that with the increase in pathological grade and malignant degree of breast cancer cells, Snail expression levels gradually increased $(\mathrm{P}<0.01)$. Western blotting revealed that miR-30a significantly inhibited Snail expression in SK-BR-3 cells, upregulated the expression of EMT-associated E-cadherin, and downregulated the expression of EMT-associated N-cadherin and Vimentin. MiR-30a was able to affect the proliferation and invasion of breast cancer cells by regulating Snail expression, and therefore has a regulatory effect on the occurrence and development of breast cancer.
\end{abstract}

\section{Introduction}

The global incidence of breast cancer has been increasing since the end of 1980s (1). According to the 'Global Cancer Report 2014' issued by the World Health Organization, there were

Correspondence to: Dr Baoqiang Xiao, Department of General Surgery, No. 254 Hospital of The People's Liberation Army, 60 Huangwei Road, Tianjin 300142, P.R. China

E-mail: xiaobaoqiang022@126.com

"Contributed equally

Key words: microRNA, breast cancer, proliferation, invasion
1.677 million new cases of breast cancer in females globally in 2012, with China accounting for $11.2 \%$ of cases (2). At present, breast cancer has the highest incidence rate in China of any malignant tumor for women (3). The majority of patients with breast cancer are the middle or late stages upon initial diagnosis, thus the curative effects are often unsatisfactory (4). The primary cause of mortality for patients with breast cancer is metastasis (5). However, the underlying molecular mechanism of breast cancer metastasis remains unresolved. Therefore, it is considered important to study and understand the underlying molecular mechanism associated with breast cancer progression and metastasis, in order to provide a scientific basis for the development of effective treatment strategies for breast cancer, and to improve the survival rate of patients with breast cancer.

In recent years, a study revealed that the occurrence and development of breast cancer is associated with the abnormal expression of microRNA (miRNA) (6). miRNA is a class of endogenous non-coding RNA. It widely exists in eukaryotic cells and has a regulatory effect on cell proliferation, apoptosis, migration and invasion (7-9). It also serves an important function in the occurrence and development of tumors (10-12). A number of studies have revealed that miR-30a is downregulated in a variety of tumor tissues, including ovarian cancer (13), lung cancer (14), hepatocellular carcinoma (15) and gastric cancer (16). Sestito et al (13) reported that the expression level of miR-30a in ovarian cancer tissue was significantly reduced, and overexpression of miR-30a in ovarian cancer cells was able to decrease the activity of phosphoinositide 3-kinase/protein kinase $\mathrm{B}$ and reduce the activation of the mitogen-activated protein kinase signaling pathway, thus inhibiting the proliferation and invasion of ovarian cancer cells, and downregulating the levels of epithelial-mesenchymal transition (EMT)-associated markers in ovarian cancer cells. Tang et al (14) revealed that miR-30a expression levels were significantly decreased in lung cancer tissues and that miR-30a expression level was closely associated with the development and prognosis of lung cancer. Liu et al (17) revealed that in hepatocellular carcinoma, miR-30a was able to regulate the expression level of Snail, which was associated with EMT, and inhibit the migration and invasion of hepatocellular carcinoma. Liu et al (18) reported that miR-30a expression was abnormal in gastric carcinoma tissue, and miR-30a was able to alter Vimentin expression by regulating the expression of runt-related transcription factor 3 , which was associated 
with EMT and therefore involved in the occurrence and development of gastric cancer.

Thus far, there are few studies on the change in miR-30a expression in breast cancer or its association with the development, proliferation and invasion of breast cancer. Therefore, in the present study, miR-30a expression in breast cancer tissue and breast cancer cell lines was detected using reverse transcription-polymerase chain reaction (RT-PCR), and miR-30a mimic and anti-miR-30a were transfected into the breast cancer cell line SK-BR-3 to observe the effects of miR-30a expression on the proliferation and invasion of SK-BR-3 cells. Zinc finger protein Snai 1, the target gene of miR-30a, was verified and the effect of miR-30a on the expression level of Snail protein was detected in order to identify a possible novel therapeutic target for the treatment of breast cancer.

\section{Materials and methods}

Clinical data. The tumor tissue and paracancerous tissue of patients with breast cancer $(n=43)$ who were treated in the No. 254 Hospital of the People's Liberation Army, (Tianjin, China) between June 2015 and February 2016 were collected. These patients were aged between 19 and 58 years old, with an mean age of $31.42 \pm 21.46$ years. Tumor tissue specimens and adjacent normal tissue specimens from patients were surgically collected. All specimens were verified as breast cancer tissues by two pathologists at No. 254 Hospital of the People's Liberation Army following surgery, while normal tissues were confirmed to have no cancer invasion. The specimens were stored in liquid nitrogen within $10 \mathrm{~min}$ of being obtained. Tumor (T) staging was defined by the length of the tumor according to the following criteria: $\mathrm{Tl} \leq 2 \mathrm{~cm} ; 2 \mathrm{~cm}<\mathrm{T} 2 \leq 5 \mathrm{~cm}$; T3>5 cm. Tumors directly invading skins and chest walls (costa, intercostal muscles, serratus anterior muscle) were defined as $\mathrm{T} 4$, without regard to their size. In total, there were 16 cases of T1, 12 cases of T2, 9 cases of T3 and 6 cases of T4. Written informed consent was obtained from all patients. The present study was approved by the ethics committee of the No. 254 Hospital of the People's Liberation Army.

Cells and reagents. Normal mammary epithelial MCF-10A cells, low invasive breast cancer MCF-7 cells, moderate metastatic and invasive breast cancer SK-BR-3 cells (19) and high metastatic and invasive human breast cancer MDA-MB-231 cells were all purchased from the Preclinical Medicine Institute of the Chinese Academy of Medical Sciences (Beijing, China). These cells were cultured in Dulbecco's modified Eagle's medium containing 10\% fetal bovine serum (Gibco; Thermo Fisher Scientific, Inc., Waltham, MA, USA). Horseradish peroxidase (HRP)-conjugated goat anti mouse immunoglobulin $\mathrm{G}$ was purchased from OriGene Technologies, Inc. (Rockville, MD, USA). The transfection reagents used were Lipofectamine 2000 (Invitrogen; Thermo Fisher Scientific, Inc.) and TRIzol ${ }^{\circledR}$ (Life Technologies; Thermo Fisher Scientific, Inc.). Negative control (NC), miRNA-30a mimic, anti-NC and anti-miRNA-30a were purchased from (Ambion; Thermo Fisher Scientific, Inc.). Boyden chambers used in the invasion assay were purchased from EMD Millipore (Billerica, MA, USA). The RT-PCR primers were synthesized by Sangon Biotech Co., Ltd. (Shanghai, China). A luciferase assay kit was purchased from Promega Corporation (Madison, WI, USA). Antibodies against Snail, E-cadherin, N-cadherin and Vimentin were purchased from Santa Cruz Biotechnology, Inc. (Dallas, TX, USA).

$R T-P C R$. Total RNA were extracted from the tissue samples using TRIzol (Invitrogen; Thermo Fisher Scientific, Inc.) cDNAs were synthesized with the Prime-Script RT reagent kit (Takara Bio, Inc., Otsu, Japan), SYBR-Green (Takara Bio, Inc., Otsu, Japan) was used as the fluorophore, according to the manufacturer's protocol. The thermocycling conditions were as follows: $95^{\circ} \mathrm{C}$ for $5 \mathrm{~min}, 95^{\circ} \mathrm{C}$ for $10 \mathrm{sec}, 60^{\circ} \mathrm{C}$ for $20 \mathrm{sec}$, for a total of 40 cycles. Primer sequences were as follows: Snail forward primer, 5'-GTGGGATGGCTGCCAGC-3'; reverse primer, 5'-TGCAGGACTCTAATCCAAGTTTAC-3'; forward primer of internal control U6, 5'-CTCGCTTCGGCAGCA CA-3'; reverse primer, 5'-AACGCTTCACGAATTTGCGT-3'. The obtained cDNA were identified by standard agarose gel electrophoresis.

Clone formation test. A total of $1 \times 10^{4}$ SK-BR-3 cells were seeded into each well of 6-well plate, and incubated overnight at $37^{\circ} \mathrm{C}$, then transfected with $\mathrm{NC}$, miR-30a mimic, anti-NC and anti-miR-30a at a concentration of $200 \mathrm{nmol} / \mathrm{l}$. Lipofectamine 2000 or Oligofectamine (Invitrogen; Thermo Fisher Scientific, Inc.) was used for transfection according to the manufacturer's protocol. At $48 \mathrm{~h}$ following transfection, cells were used for further experiments. Primer sequences were as follows: NC sense, 5'-UUCUCCGAACGUGUCACG UTT-3' and antisense, 5'-ACGUGACACGUUCGGAGA ATT-3'; miR-30a mimic sense, 5'-UGUAAACAUCCUCGA CUGGAAG-3' and antisense, 5'-UCCAGUCGAGGAUGU UUACAUU-3'; anti-NC, 5'-CAGUACUUUUGUGUAGUA CAA-3'; anti-miR-30a, 5'-CUUCCAGUCGAGGAUGUUUAC A-3'. The mimic was purchased from Guangzhou RiboBio Co., Ltd. (Guangzhou, China). Cells were inoculated in six-well plates at a density of $5 \times 10^{3}$ cells per well, and cultured for $72 \mathrm{~h}$. The formed clones were fixed with $70 \%$ methanol at $4{ }^{\circ} \mathrm{C}$ overnight, and stained with $20 \%$ methanol containing $0.1 \%$ crystal violet for $30 \mathrm{~min}$ at $37^{\circ} \mathrm{C}$. Colonies that contained $>50$ cells were counted under a light microscope (magnification, x100; Eclipse TiU, Nikon Corporation., Tokyo, Japan).

Invasion test. The invasive ability of cells was detected using Boyden chambers. The cells used in this experiment were stained with $0.1 \%$ trypan blue (Beijing Solarbio Science and Technology Co., Ltd., Beijing, China) for $5 \mathrm{~min}$ at room temperature counted under the a light microscope (magnification, x200) (Eclipse TiU; Nikon Corporation) in 5 randomly selected fields, and then suspended. A total of $1 \times 10^{5}$ cells were placed in the upper chamber. Matrigel (5-10 $\mu \mathrm{g}$ in $50 \mu \mathrm{l}$ per well) was pre-coated in a 24 -well plate, and dried overnight in a laminar airflow hood and serum-free medium (StemPro ${ }^{\circledR}$ MSC SFM; Invitrogen: Thermo Fisher Scientific, Inc.) was added up to $300 \mu 1$. A total of $500 \mu 1$ serum-free medium (StemPro ${ }^{\circledR}$ MSC SFM; Invitrogen: Thermo Fisher Scientific, Inc.) supplemented with $10 \%$ fetal bovine serum (Thermo Fisher Scientific Inc.) was added to the lower chamber. After $12 \mathrm{~h}$ of incubation, the non-invasive cells were removed from the upper chamber with a cotton swab. 
A
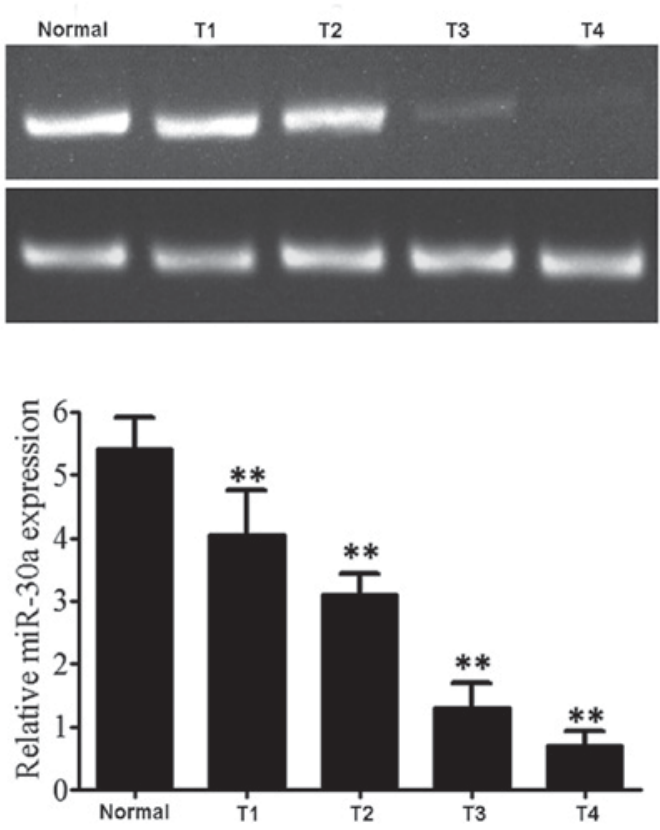

B
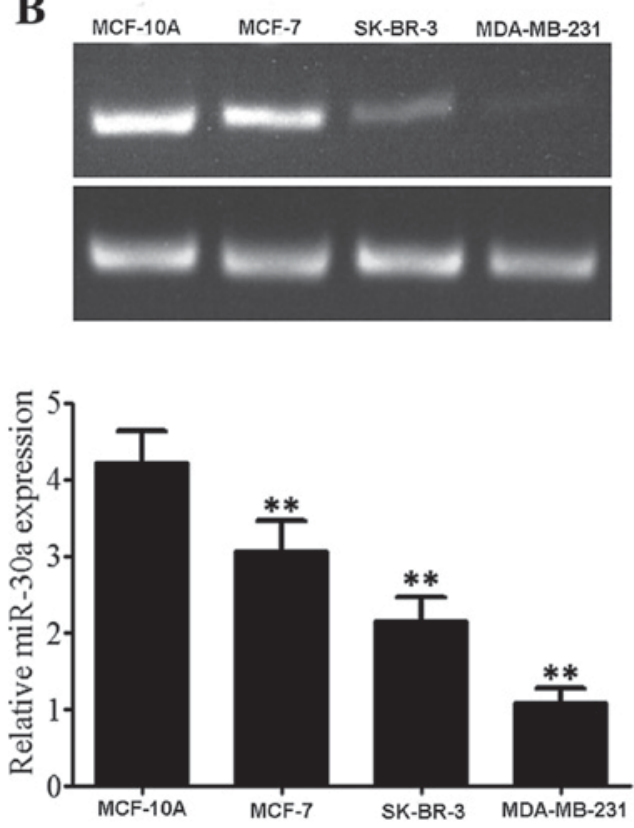

Figure 1. The expression of miR-30a mRNA in breast cancer tissues and cells. (A) miR-30a mRNA expression in breast cancer tissues: 1, Paracancerous tissue; 2, T1; 3, T2; 4, T3; 5, T4; (B) miR-30a expression in breast cancer cells, 1, MCF-10A cells; 2, MCF-7 cells; 3, SK-BR-3 cells; 4, MDA-MB-231 cells; ${ }^{* *} \mathrm{P}<0.01$ vs. negative control. miRNA, microRNA.

The cells in the lower chamber were fixed with $5 \%$ glutaraldehyde for $5 \mathrm{~min}$ at $4^{\circ} \mathrm{C}$ and stained with $0.1 \%$ crystal violet for $30 \mathrm{~min}$ at $37^{\circ} \mathrm{C}$. The number of invaded cells was counted under the microscope (x200 magnification) (Eclipse TiU, Nikon Corporation) in 5 randomly selected fields.

Forecasting possible target genes of miR-30a using bioinformatics methods. According to Sequence complementarity (the 706-713 site in the 3'untranslated region (3'UTR) of Snail mRNA was a possible site of action of miR-30a), Snail was selected as the target gene of miR-30a using the target gene prediction software TargetScans (http://www.targetscan. org/vert_71/).

Verification of target gene by luciferase reporter gene test. The renilla luciferase gene with the 3'UTR was PCR-amplified from the pRL-SV40 plasmid (Promega Corporation) and inserted into the 3'UTR site of pMIR-REPORT. Luciferase expression constructs were generated by cloning of the 3'UTRs of Snail into the modified pMIR vector (pMIR-RL). Amplified 3' UTR were cloned downstream of the firefly luciferase coding region in the pMIR-REPORTTM (Ambion; Thermo Fisher Scientific, Inc.). The pMIR-luciferase reporter vectors (100 ng) were transfected into SK-BR-3 cells in 6-well plates together with miR-30a oligonucleotides (100 $\mathrm{nM}$ ) by using Lipofectamine 2000. After $72 \mathrm{~h}$ of culture, luciferase activity was detected with Dual-Luciferase Reporter assay system (Promega Corporation) according to the manufacturer's protocol. Firefly luciferase activity was normalized to that of Renilla luciferase.

Western blot analysis. The protein concentration was determined by one method of quantification (LI-COR Biosciences, Lincoln, NE, USA), a total of $50 \mu \mathrm{g}$ protein was loaded in to each well and were separated on $8 \%$ SDS-PAGE and $5 \%$ concentrated gel, then transferred to polyvinylidene difluoride membranes in a half-dry state and blocked at room temperature for $2 \mathrm{~h}$ with Tris-Buffered Saline and Tween-20 (TBST) containing 5\% bovine serum albumin (BSA, Abcam, Cambridge, UK). The primary antibody (1:800; rabbit polyclonal anti-iNOS, sc-650; Santa Cruz Biotechnology) was added and incubated at $4^{\circ} \mathrm{C}$ overnight with agitation. The membrane was washed with $0.1 \%$ TBST three times, for $5 \mathrm{~min}$ each time. HRP-conjugated secondary antibody $(1: 10,000$; goat anti-rabbit IgG (H+L)-HRP conjugate, cat. no. 170-6515, Bio-Rad Laboratories, Inc.) was added and incubated at room temperature for $1 \mathrm{~h}$. Following a wash with $0.1 \%$ TBST, the bands on the membrane were colored with Supersignal West Femto HRP-sensitive chemiluminescence substrate (Pierce; Thermo Fisher Scientific, Inc.). Actin was used as an internal control. All the experiments were repeated at least three times. The immunoreactive proteins were detected using the ECL Plus Western Blotting Detection system (GE Healthcare, Chicago, IL, USA), visualized by Molecular Imager ${ }^{\circledR}$ VersaDoc $^{\text {TM }}$ MP Imaging systems (Bio-Rad Laboratories, Hercules, CA, USA) and the densitometry were measured by the professional image analysis software, ImagePro Plus 6.0 (IPP6.0, Media Cybernetics, Bethesda, USA).

Statistical analysis. Statistical analysis was conducted using SPSS software (version 17.0; SPSS, Inc., Chicago, IL, USA). Measurement data are expressed as mean \pm standard deviation. Comparisons between data in two groups were conducted using Student's t-test. Multi-group comparisons were conducted using univariate analysis of variance. Inter-group comparisons were conducted using Student-Newman-Keuls (SNK) method. $\mathrm{P}<0.05$ was considered to indicate a statistically significant difference. 

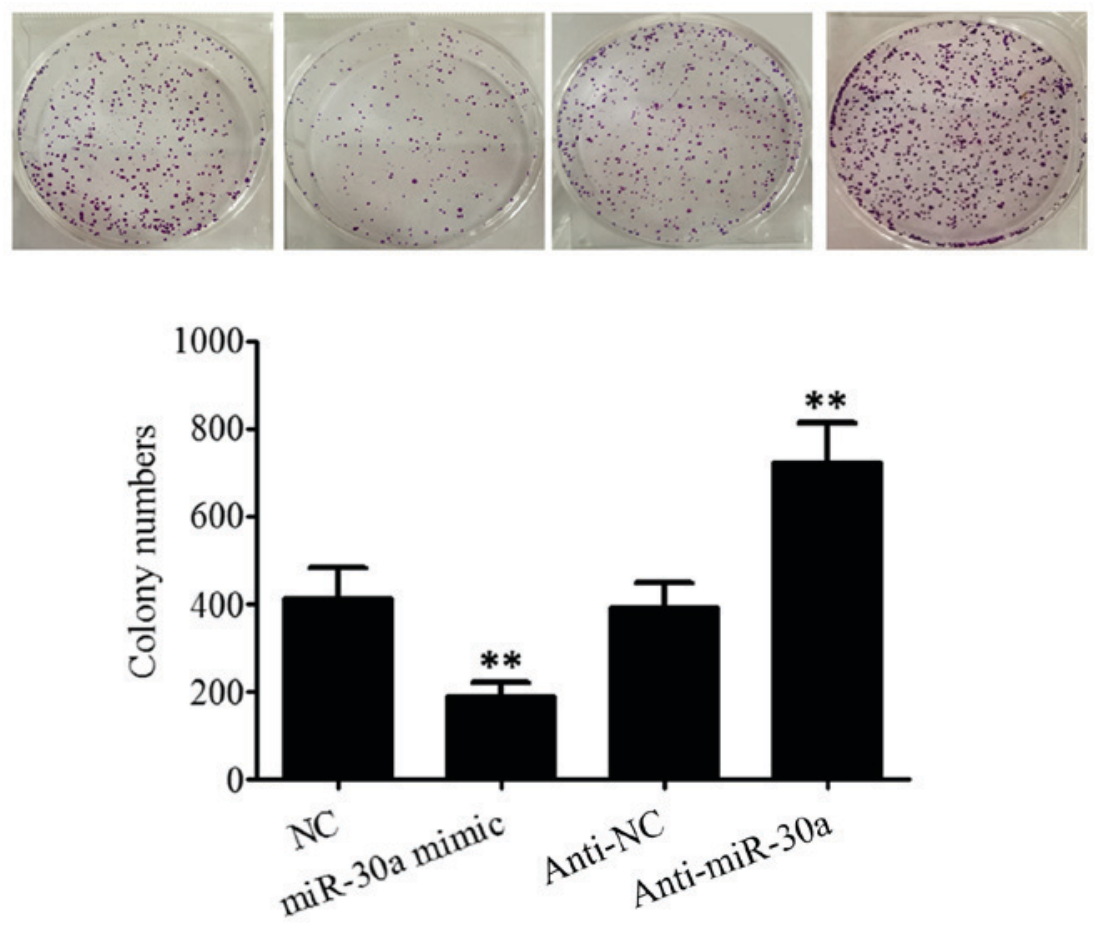

Figure 2. Effect of miR-30a on the proliferation of breast cancer cells detected by clone formation test ${ }^{* *} \mathrm{P}<0.01 \mathrm{vs}$. negative control. miR, microRNA; NC, negative control.

\section{Results}

RT-PCR.MiR-30a expression levels in breast cancer tissues and cell lines are demonstrated by the RT-PCR results (Fig. 1A). The relative expression level of miR-30a was $5.43 \pm 0.51$ in normal tissues; $4.06 \pm 0.69$ in stage $\mathrm{T} 1$ breast cancer tissues; $3.11 \pm 0.33$ in stage T2 breast cancer tissues; $1.32 \pm 0.39$ in stage $\mathrm{T} 3$ breast cancer tissues; and $0.71 \pm 0.23$ in stage T4 breast cancer tissues. With the increase of pathological grade of breast cancer, miR-30a expression level gradually decreased $(\mathrm{F}=223.806, \mathrm{P}<0.001)$. As presented in Fig. 1B, the results of RT-PCR revealed that the relative expression level of miR-30a was $4.22 \pm 0.41$ in normal mammary epithelial cell MCF-10A; $3.07 \pm 0.39$ in the low invasive breast cancer cell line MCF-7; $2.16 \pm 0.31$ in the moderately metastatic and invasive breast cancer cell line SK-BR-3; and 1.08 \pm 0.19 in highly metastatic and invasive human breast cancer cell line MDA-MB-231. With the increase of the malignant degree of breast cancer cells, expression level of $\mathrm{miR}-30 \mathrm{a}$ decreased $(\mathrm{F}=47.260$, $\mathrm{P}<0.001)$.

Effect of miR-30a on the proliferation of breast cancer cells. The effect of miR-30a on the proliferation of SK-BR-3 breast cancer cells was detected using a clone formation test. The results (Fig. 2) revealed that the number of clones of SK-BR-3 cells was $415.63 \pm 69.22$ in the NC group; $189.74 \pm 31.37$ in the miR-30a mimic group; $393.68 \pm 57.16$ in the anti-NC group; and $725.82 \pm 89.57$ in the anti-miR-30a group. Transfection with miR-30a mimic significantly inhibited the proliferation of SK-BR-3 cells ( $\mathrm{t}=5.148, \mathrm{P}=0.003)$, while transfection with anti-miR-30a significantly increased the proliferation of SK-BR-3 cells ( $\mathrm{t}=5.414, \mathrm{P}=0.003)$. This suggests that, miR-30a was able to inhibit the proliferation of SK-BR-3 breast cancer cells. It was also identified that, transfection with miR-30a inhibited the proliferation of MDA-MB-231 human breast cancer cells which exhibit high invasive and metastatic abilities. Inhibiting miR-30a expression was observed to promote the proliferation of breast cancer MCF-7 cells which exhibit a low invasive ability (data not shown).

Effect of miR-30a on the invasive ability of breast cancer cells. As presented in Fig. 3, the results of the cell invasion assay revealed that the quantity of invaded SK-BR-3 cells was $102.6 \pm 28.5$ in the NC group, 55.7 \pm 13.6 in the miR-30a mimic group, $105.5 \pm 25.9$ in the anti-NC group and $171.2 \pm 27.2$ in the anti-miR-30a group. Transfection with miR-30a mimic significantly inhibited the invasive ability of SK-BR-3 cells $(\mathrm{t}=2.572$, $\mathrm{P}=0.031$ ), while transfection with anti-miR-30a significantly increased the invasive ability of SK-BR-3 cells $(\mathrm{t}=2.989$, $\mathrm{P}<0.020)$. This suggests that miR-30a was able to inhibit the invasion of SK-BR-3 cells. The results of the present study suggest that transfecting miR-30a may inhibit the invasiveness of MDA-MB-231 cells which exhibit high invasive and metastatic abilities. Furthermore, inhibition of miR-30a expression promoted the invasion of breast cancer MCF-7 cells which exhibit low invasive ability (data not shown).

Forecasting miR-30a target gene by TargetScan software. The target gene prediction software TargetScans indicated that Snail may be the target gene of miR-30a. The predicted binding site of miR-30a, Snail 3'-UTR, is presented in Fig. 4A. The results of a luciferase reporter gene assay revealed that following co-transfection with wild-type pMIR-Snail 3'-UTR vector and miR-30a, luciferase activity was significantly reduced $(\mathrm{t}=10.337, \mathrm{P}<0.001)$, however, following co-transfection with mutant pMIR-Snail 3'-UTR vector and miR-30a, luciferase 

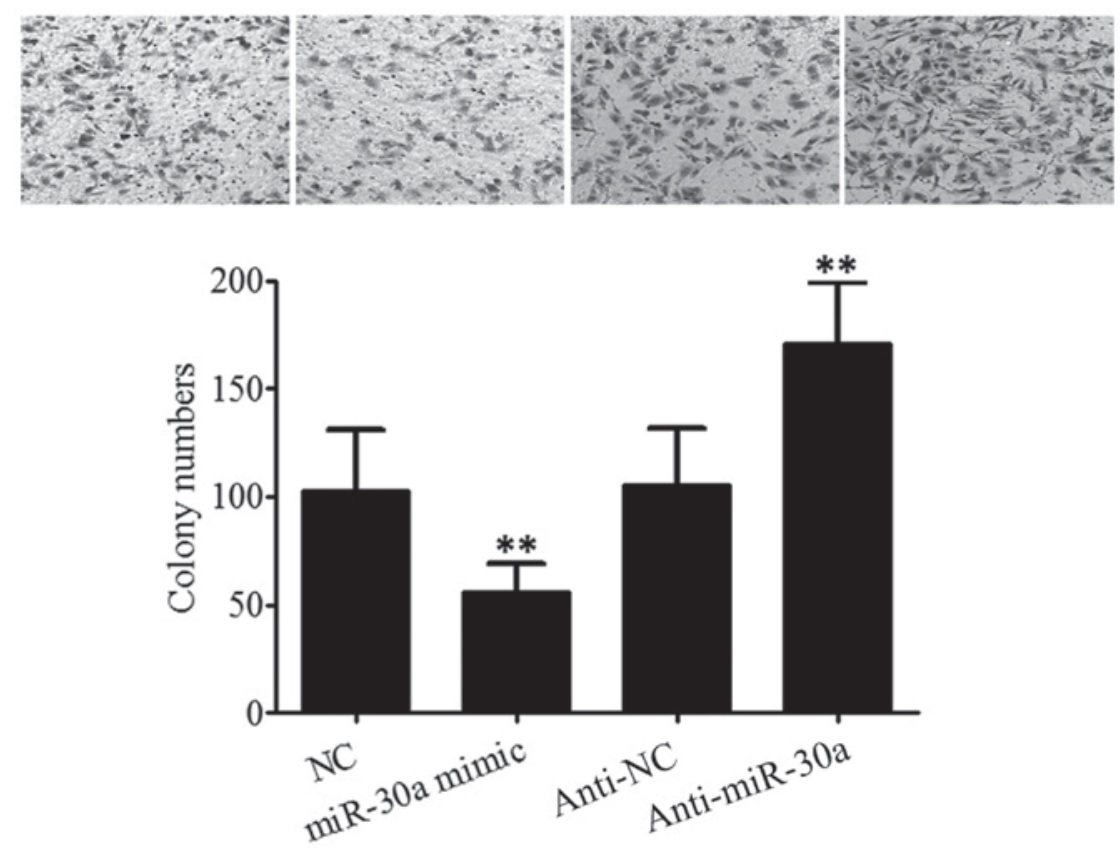

Figure 3. Effect of miR-30a on the invasion of breast cancer cells ${ }^{* *} \mathrm{P}<0.01$ vs. negative control. miR, microRNA; NC, negative control.

$\mathbf{A}$

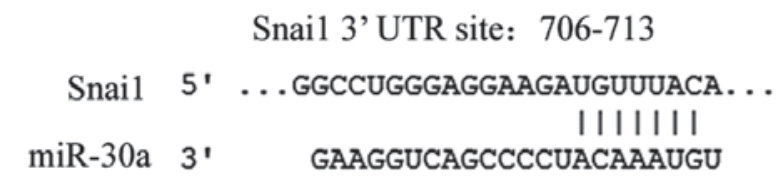

B

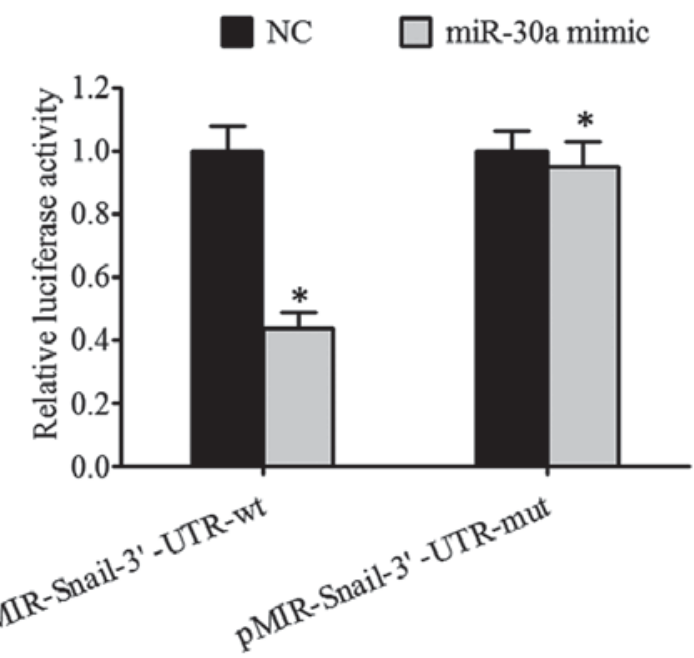

Figure 4. Forecasting miR-30a target gene using the target gene prediction software TargetScans. (A) Predicted miR-30a binding target site of Snail 3'-UTR. (B) Verification of the interaction between miR-30a and Snail ${ }^{* *} \mathrm{P}<0.01$ vs. negative control. miR, microRNA; NC, negative control.

activity did not change significantly. The luciferase activity did not significantly change $(\mathrm{P}>0.05)$ following co-transfection of wild type pMIR- Snail 3'-UTR vector and miR-30b, miR-30c, miR-30d and miR-30e. This suggests that there is a direct interaction between miR-30a and Snail (Fig. 4B).

Snail expression in breast cancer tissues and cell lines. The results of western blotting (Fig. 5A) revealed that the relative expression level of Snail was $1.00 \pm 0.08$ in normal tissues, $1.65 \pm 0.19$ in stage $\mathrm{T} 1$ breast cancer tissues, $2.13 \pm 0.33$ in stage $\mathrm{T} 2$ breast cancer tissues, $2.71 \pm 0.29$ in stage $\mathrm{T} 3$ breast cancer tissues and 3.30 \pm 0.18 in stage T4 breast cancer tissues. An increase in the expression level of Snail was associated with increased tumor grade $(\mathrm{F}=372.022, \mathrm{P}<0.001)$. The results of western blotting (Fig. 5B) revealed that the relative expression level of Snail was $1.00 \pm 0.13$ in normal mammary epithelial cells MCF-10A, 1.82 \pm 0.21 in low invasive breast cancer cells MCF-7, 2.61 \pm 0.19 in moderately metastatic and invasive breast cancer cells SK-BR-3 and 3.25 \pm 0.35 in highly metastatic and invasive human breast cancer cells MDA-MB-231. Snail expression levels were associated with increased levels of malignancy $(\mathrm{F}=51.938, \mathrm{P}<0.001)$.

Effects ofmiR-30a on the expressionlevels of Snail,E-cadherin, $N$-cadherin and Vimentin proteins in breast cancer cells. As presented in Fig. 6, miR-30a was able to significantly inhibit Snail expression in SK-BR-3 cells, upregulate the expression of EMT-associated E-cadherin, and downregulate the expression of EMT-associated N-cadherin and Vimentin.

\section{Discussion}

Previous studies revealed that a variety of miRNAs, including miR-224, miR-21 and miR-320, were abnormally expressed in breast cancer (20-22). The expression levels of miR-224 in breast cancer tissues and breast cancer cell lines were decreased, and their decreased level was associated with the degree of malignancy of the breast cancer. miR-224 has been demonstrated to regulate the proliferative, migratory and invasive abilities of breast cancer cells by directly targeting fizzled 5 (20). The expression level of miR-21 in breast cancer tissue was observed to be abnormal (23). Following overexpression of miR-21, the proliferative and invasive abilities of breast cancer cells significantly increased (24). Furthermore, a study 
A
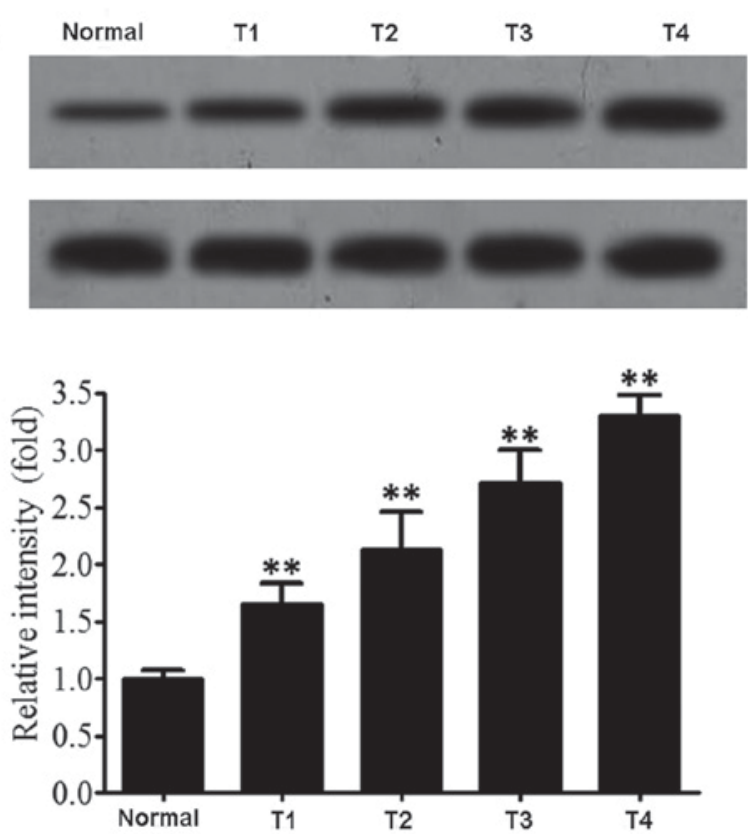

B
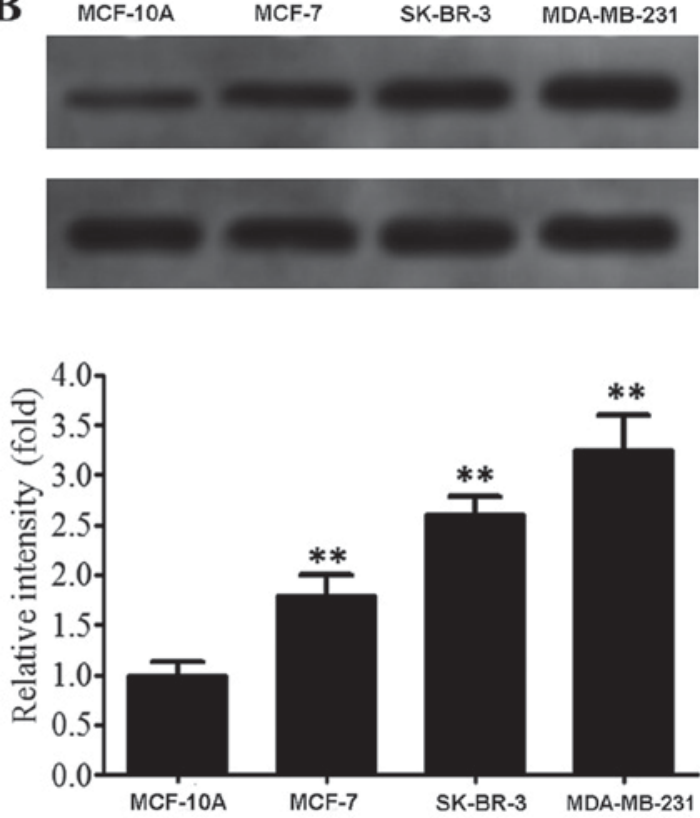

Figure 5. Snail expression in breast cancer tissues and cells. (A) Snail expression in breast cancer tissues: 1, paracancerous tissue; 2, T1; 3, T2; 4, T3; 5, T4; ${ }^{* *} \mathrm{P}<0.01$. (B) Snail expression in breast cancer cells: $1, \mathrm{MCF}-10 \mathrm{~A}$ cells; 2 , MCF-7 cells: 3 , SK-BR-3 cells; 4 , MDA-MB-231 cells. ${ }^{* *} \mathrm{P}<0.01$ vs. negative control. miR, microRNA.

A

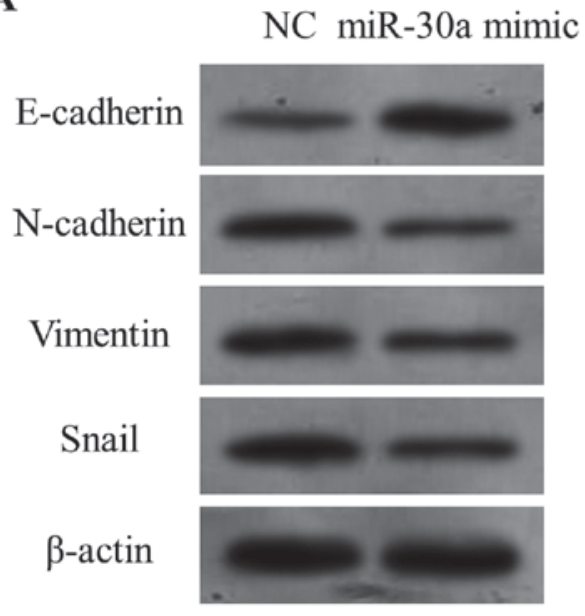

B

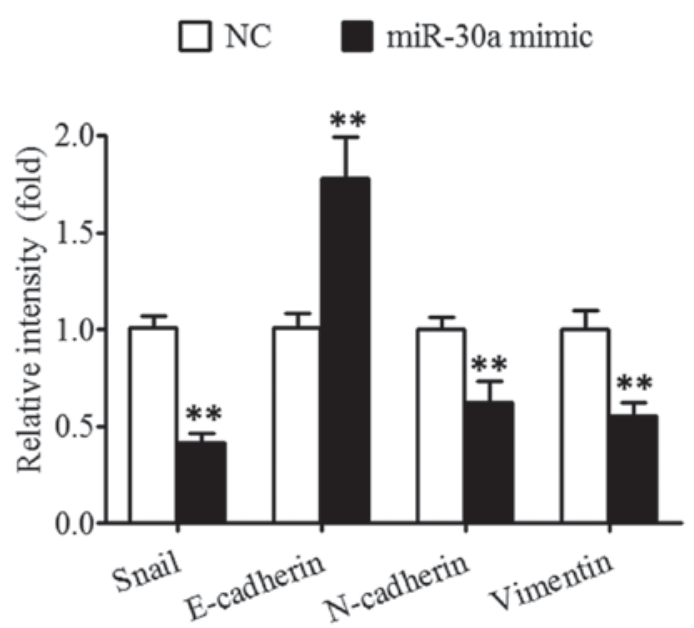

Figure 6. Effects of miR-30a on the expression levels of Snail, E-cadherin, $\mathrm{N}$-cadherin and vimentin proteins in breast cancer cells. ${ }^{* *} \mathrm{P}<0.01$ vs. negative control. miR, microRNA; NC, negative control.

confirmed that miR-21-3p functioned primarily by targeting multiple downstream target genes, including programed cell death protein 4, Fas Ligand, phosphatase and tensin homolog, Ras homolog family member B, Maspin, tissue inhibitor of metalloproteinases 3 and reversion inducing cysteine rich protein with kazal motifs (21). MiR-320 was able to regulate the invasive ability of breast cancer cells by regulating the expression of the downstream target gene metadherin and inhibiting breast cancer (22). In summary, a number of miRNAs may affect the occurrence, development, invasion and metastasis of breast cancer via targeted regulation of downstream genes. A number of studies revealed that miR-30a has a regulatory effect in ovarian cancer, lung cancer, liver cancer and other tumors $(12-14,17,18)$. Therefore, the present study analyzed the expression of miR-30a in breast cancer and explored the potential molecular mechanism by which it regulates the proliferation and invasion of breast cancer cells.

The aforementioned miRNAs are closely associated with the occurrence and development of breast cancer. The present study focused on the role of miR-30a in the proliferation and invasion of breast cancer, and explored the mechanism by which it regulates the proliferation and invasion of breast cancer cells. In the present study, miR-30a expression in breast cancer tissues and breast cancer cells was first detected using RT-PCR. The results revealed that with as the pathological grade and degree of malignancy of breast cancer cells increased, the expression level of miR-30a decreased. The effect of miR-30a on the proliferation and invasion of 
breast cancer cell lines was further evaluated. The results revealed that transfection with miR-30a was able to significantly decrease the colony formation and invasive ability of SK-BR-3 cells, while transfection with anti-miR-30a significantly increased the colony formation and invasive ability of SK-BR-3 cells. This suggests that miR-30a may inhibit breast cancer by inhibiting the proliferation and invasion of breast cancer cells. Additional data gathered in the present study identified that miR-30a was able to inhibit the proliferation and invasion of MDA-MB-231 human breast cancer cells, which have high invasive and metastatic abilities, while inhibiting miR-30a expression promoted the proliferation and invasion of MCF-7 breast cancer cells which have a low invasive ability. RT-PCR revealed that the expression level of miR-30a in breast cancer tissue was significantly decreased compared with the normal control tissues. The authors hypothesize that the decrease in miR-30a in breast cancer tissue may increase the proliferative and invasive abilities of breast cancer cells, thus leading to the occurrence and development of breast cancer.

miRNAs serve a biological function by regulating downstream target genes. Different functions of downstream target genes may lead to different functions of the same miRNA in different tumor tissues (25). This study confirmed that miR-30a had an inhibitiory effect on the occurrence of breast cancer. In order to evaluate the downstream target genes of miR-30a which are able to inhibit tumor development in breast cancer tissue, the target gene of miR-30a was screened for using the target gene prediction software TargetScans. Among the numerous potential target genes, due to its function in the development of human cancer (26-29), Snail was selected as the target gene of interest. Snail is a regulatory factor in the process of EMT in tumor cells (30). It promotes the occurrence of EMT in tumor cells by upregulating Vimentin and downregulating the expression of E-cadherin. Its abnormal expression is closely associated with the invasion, metastasis and recurrence of tumor cells (31). In the present study, the results of a luciferase reporter gene assay confirmed that following cotransfection with wild-type pMIR-Snail13-UTR' vector and miR-30a, the luciferase activity was significantly reduced, while following cotransfection with mutant pMIR-Snail13-UTR' vector and miR-30a, luciferase activity did not significantly change. The luciferase activity did not significantly change $(\mathrm{P}>0.05)$ following cotransfection with wild type pMIR-Snai1 3'-UTR vector and miR-30b. The data indicates a direct interaction between miR-30a and Snail. Snail may be a target gene regulated by miR-30a. Therefore, miR-30a may serve a function inhibiting breast cancer by regulating the expression of Snail. Therefore further study was undertaken. First, the expression of Snail in breast cancer tissues and breast cancer cells was detected. The results revealed that with the increase in pathological grade and malignant degree of breast cancer cells, Snail protein expression level gradually increased $(\mathrm{P}<0.01)$. The effects of miR-30a on the expression levels of Snail, E-cadherin, $\mathrm{N}$-cadherin and Vimentin proteins in breast cancer cells were further evaluated. The results revealed that miR-30a was able to significantly inhibit the expression of Snail in SK-BR-3 cells, upregulate the expression of EMT-associated E-cadherin, and downregulate the expression of EMT-associated N-cadherin and Vimentin. These results suggest that Snail is a downstream target gene of miR-30a, and that miR-30a may inhibit breast cancer by regulating the expression of Snail in breast cancer tissues.

In summary, the present study has revealed that the expression of miR-30a in breast cancer tissue was significantly decreased, and was closely associated with the malignant degree of breast cancer. miR-30a is able to inhibit the proliferation and invasion of breast cancer cells. A decrease in miR-30a expression levels in breast cancer tissue may be associated with the occurrence and development of breast cancer. Further study revealed that miR-30a inhibited the proliferation and invasion of breast cancer cells by regulating the expression of Snail, E-cadherin, $\mathrm{N}$-cadherin and Vimentin proteins.

\section{Acknowledgements}

Not applicable.

\section{Funding}

No funding received.

\section{Availability of data and materials}

The datasets used and/or analyzed during the current study are available from the corresponding author on reasonable request.

\section{Authors' contributions}

BX designed the experiments. XS performed the bioinformatic analyses. All other experiments were performed by JB. The figures were prepared by XS, and the manuscript was written by JB, with input from BX and XS.

\section{Ethics approval and consent to participate}

The present study was approved by the ethics committee of the No. 254 Hospital of the People's Liberation Army. Written informed consent was obtained from all patients.

\section{Patient consent for publication}

All study participants provided consent for the publication of data.

\section{Competing interests}

The authors declare that they have no competing interests.

\section{References}

1. DeSantis C, Ma J, Bryan L and Jemal A: Breast cancer statistics, 2013. CA Cancer J Clin 64: 52-62, 2014.

2. McGuire S: World Cancer Report 2014. Geneva, Switzerland: World Health Organization, International agency for research on cancer, WHO Press, 2015. Adv Nutr 7: 418-419, 2016.

3. Fan L, Strasser-Weippl K, Li JJ, St Louis J, Finkelstein DM, Yu KD, Chen WQ, Shao ZM and Goss PE: Breast cancer in China. Lancet Oncol 15: e279-e289, 2014

4. Dickens C, Joffe M, Jacobson J, Venter F, Schüz J, Cubasch H and McCormack V: Stage at breast cancer diagnosis and distance from diagnostic hospital in a periurban setting: A South African public hospital case series of over 1,000 women. Int J Cancer 135: 2173-2182, 2014. 
5. Narod SA, Iqbal J, Giannakeas V, Sopik V and Sun P: Breast cancer mortality after a diagnosis of ductal carcinoma in situ. JAMA Oncol 1: 888-896, 2015.

6. Xia M, Li H, Wang JJ,Zeng HJ and Wang SH: MiR-99a suppress proliferation, migration and invasion through regulating insulin-like growth factor 1 receptor in breast cancer. Eur Rev Med Pharmacol Sci 20: 1755-1763, 2016.

7. Calin GA, Sevignani C, Dumitru CD, Hyslop T, Noch E, Yendamuri S, Shimizu M, Rattan S, Bullrich F, Negrini M and Croce CM: Human microRNA genes are frequently located at fragile sites and genomic regions involved in cancers. Proc Nat Acad Sci USA 101: 2999-3004, 2004.

8. Rathod SS, Rani SB, Khan M, Muzumdar D and Shiras A: Tumor suppressive miRNA-34a suppresses cell proliferation and tumor growth of glioma stem cells by targeting Akt and Wnt signaling pathways. FEBS Open Bio 4: 485-495, 2014

9. Li XT, Wang HZ, Wu ZW, Yang TQ, Zhao ZH, Chen GL, Xie XS, Li B, Wei YX, Huang YL, et al: miR-494-3p regulates cellular proliferation, invasion, migration, and apoptosis by PTEN/AKT signaling in human glioblastoma cells. Cell Mol Neurobiol 35: 679-687, 2015

10. Ozen M, Creighton CJ, Ozdemir M and Ittmann M: Widespread deregulation of microRNA expression in human prostate cancer. Oncogene 27: 1788-1793, 2008.

11. Volinia S, Calin GA, Liu CG, Ambs S, Cimmino A, Petrocca F, Visone R, Iorio M, Roldo C, Ferracin M, et al: A microRNA expression signature of human solid tumors defines cancer gene targets. Proc Natl Acad Sci USA 103: 2257-2261, 2006.

12. Liu H, Wang Y, Li X, Zhang YJ, Li J, Zheng YQ, Liu M, Song X and Li XR: Expression and regulatory function of miRNA-182 in triple-negative breast cancer cells through its targeting of profilin 1. Tumour Biol 34: 1713-1722, 2013.

13. Sestito R, Cianfrocca R, Rosanò L, Tocci P, Semprucci E, Di Castro V, Caprara V, Ferrandian G, Sacconi A, Blandino G and Bagnato A: MiR-30a inhibits endothelin A receptor and chemoresistance in ovarian carcinoma. Oncotarget 7: 4009-4023; 2016.

14. Tang R, Liang L, Luo DZ, Feng ZB, Huang QX, He RQ, Gan TQ Yang LH and Chen G: Downregulation of MiR-30a is associated with poor prognosis in lung cancer. Med Sci Monit 21:2514-2520, 2015.

15. Wang W, Lin H, Zhou L, Zhu Q, Gao S, Xie H, Liu Z, Xu Z, Wei J, Huang $X$ and Zheng S: MicroRNA-30a-3p inhibits tumor proliferation, invasiveness and metastasis and is downregulated in hepatocellular carcinoma. Eur J Surg Oncol 40: 1586-1594, 2014.

16. Wang LL, Zhang XH, Zhang X and Chu JK: MiR-30a increases cisplatin sensitivity of gastric cancer cells through suppressing epithelial-to-mesenchymal transition (EMT). Eur Rev Med Pharmacol Sci 20: 1733-1739, 2016.

17. Liu Z, Tu K and Liu Q: Effects of microRNA-30a on migration, invasion and prognosis of hepatocellular carcinoma. FEBS Lett 588: 3089-3097, 2014

18. Liu Z, Chen L, Zhang X, Xu X, Xing H, Zhang Y, Li W, Yu H, Zeng J and Jia J: RUNX3 regulates vimentin expression via miR-30a during epithelial-mesenchymal transition in gastric cancer cells. J Cell Mol Med 18: 610-623, 2014.
19. Wang Y, Li SJ, Pan J, Che Y, Yin J and Zhao Q: Specific expression of the human voltage-gated proton channel Hv1 in highly metastatic breast cancer cells, promotes tumor progression and metastasis. Biochem Biophys Res Commun 412: 353-359, 2011.

20. Liu F, Liu Y, Shen J, Zhang G and Han J: MicroRNA-224 inhibits proliferation and migration of breast cancer cells by down-regulating fizzled 5 expression. Oncotarget 7: 49130-49142, 2016.

21. Kuang Y and Nie YJ: Exploration of the regulatory effect of miR-21 on breast cancer cell line proliferation and invasion as well as the downstream target genes. Asian Pac J Trop Med 9: 470-473, 2016

22. Yu J, Wang JG, Zhang L, Yang HP, Wang L, Ding D, Chen Q, Yang WL, Ren KH, Zhou DM, et al: MicroRNA-320a inhibits breast cancer metastasis by targeting metadherin. Oncotarget 7: 38612-38625, 2016.

23. Yan LX, Huang XF, Shao Q, Huang MY, Deng L, Wu QL, Zeng YX and Shao JY: MicroRNA miR-21 overexpression in human breast cancer is associated with advanced clinical stage, lymph node metastasis and patient poor prognosis. RNA 14: 2348-2360, 2008

24. Yan LX, Wu QN, Zhang Y, Li YY, Liao DZ, Hou JH, Fu J, Zeng MS, Yun JP, Wu QL, et al: Knockdown of miR-21 in human breast cancer cell lines inhibits proliferation, in vitro migration and in vivo tumor growth. Breast Cancer Res 13: R2, 2011.

25. Crow MS, Lum KK, Sheng X, Song B and Cristea IM: Diverse mechanisms evolved by DNA viruses to inhibit early host defenses. Crit Rev Biochem Mol Biol 51: 452-481, 2016.

26. Qi J, Li T, Bian H, Li F, Ju Y, Gao S, Su J, Ren W and Qin C: SNAI1 promotes the development of HCC through the enhancement of proliferation and inhibition of apoptosis. FEBS Open Bio 6: 326-337, 2016.

27. Yang L, Wang YJ, Zheng LY, Jia YM, Chen YL, Chen L, Liu DG, Li XH, Guo HY, Sun YL, et al: Genetic polymorphisms of TGFB1, TGFBR1, SNAI1 and TWIST1 are associated with endometrial cancer susceptibility in Chinese Han women. PLoS One 11: e0155270, 2016

28. Haraguchi M, Sato M and Ozawa M: CRISPR/Cas9n-mediated deletion of the Snail 1Gene (SNAI1) reveals its role in regulating cell morphology, cell-cell interactions, and gene expression in ovarian cancer (RMG-1) cells. PLoS One 10: e0132260, 2015.

29. Bai Z, Sun J, Wang X, Wang H, Pei H and Zhang Z: MicroRNA-153 is a prognostic marker and inhibits cell migration and invasion by targeting SNAI1 in human pancreatic ductal adenocarcinoma. Oncol Rep 34: 595-602, 2015.

30. Wang Y, Shi J, Chai K, Ying X and Zhou BP: The role of Snail in EMT and tumorigenesis. Curr Cancer Drug Targets 13: 963-972, 2013.

31. Kaufhold S and Bonavida B: Central role of Snail1 in the regulation of EMT and resistance in cancer: A target for therapeutic intervention. J Exp Clin Cancer Res 33: 62, 2014. 